Trinity University

Digital Commons @ Trinity

Psychology Faculty Research

Psychology Department

7-2015

\title{
Comparative Analysis of Meissner's Corpuscles in the Fingertips of Primates
}

\author{
A. Verendeev \\ C. Thomas \\ S. C. McFarlin \\ William D. Hopkins \\ Kimberley A. Phillips \\ Trinity University, kphilli1@trinity.edu
}

See next page for additional authors

Follow this and additional works at: https://digitalcommons.trinity.edu/psych_faculty

Part of the Psychology Commons

Publication Details

Journal of Anatomy

\section{Repository Citation}

Verendeev, A., Thomas, C., McFarlin, S. C., Hopkins, W. D., Phillips, K. A., \& Sherwood, C. C. (2015).

Comparative analysis of Meissner's corpuscles in the fingertips of primates. Journal of Anatomy, 227(1), 72-80. doi: $10.1111 /$ joa. 12327

This Post-Print is brought to you for free and open access by the Psychology Department at Digital Commons @ Trinity. It has been accepted for inclusion in Psychology Faculty Research by an authorized administrator of Digital Commons@ Trinity. For more information, please contact jcostanz@trinity.edu. 
Authors

A. Verendeev, C. Thomas, S. C. McFarlin, William D. Hopkins, Kimberley A. Phillips, and Chet C. Sherwood 


\title{
Comparative analysis of Meissner's corpuscles in the fingertips of primates
}

\author{
Andrey Verendeev, ${ }^{1}$ Christian Thomas, ${ }^{1}$ Shannon C. McFarlin, ${ }^{1}$ William D. Hopkins, ${ }^{2,3}$ \\ Kimberley A. Phillips ${ }^{4}$ and Chet C. Sherwood ${ }^{1}$ \\ ${ }^{1}$ Department of Anthropology, Center for the Advanced Study of Human Paleobiology, The George Washington University, \\ Washington, DC, USA \\ ${ }^{2}$ Neuroscience Institute and Language Center, Georgia State University, Atlanta, GA, USA \\ ${ }^{3}$ Division of Developmental and Cognitive Neuroscience, Yerkes National Primate Research Center, Atlanta, GA, USA \\ ${ }^{4}$ Department of Psychology, Trinity University, San Antonio, TX, USA
}

\begin{abstract}
Meissner's corpuscles (MCs) are tactile mechanoreceptors found in the glabrous skin of primates, including fingertips. These receptors are characterized by sensitivity to light touch, and therefore might be associated with the evolution of manipulative abilities of the hands in primates. We examined MCs in different primate species, including common marmoset (Callithrix jacchus, $n=5$ ), baboon (Papio anubis, $n=2$ ), rhesus macaque (Macaca mulatta, $n=3$ ), chimpanzee (Pan troglodytes, $n=3$ ), bonobo (Pan paniscus, $n=1$ ) and human (Homo sapiens, $n=8$ ). Fingertips of the first, second and fourth digits were collected from both hands of specimens, dissected and histologically stained using hematoxylin and eosin. The density (MCs per $1 \mathrm{~mm}^{2}$ ) and the size (cross-sectional diameter of MCs) were quantified. Overall, there were no differences in the densities of MCs or their size among the digits or between the hands for any species examined. However, MCs varied across species. We found a trend for higher densities of MCs in macaques and humans compared with chimpanzees and bonobos; moreover, apes had larger MCs than monkeys. We further examined whether the density or size of MCs varied as a function of body mass, measures of dexterity and dietary frugivory. Among these variables, only body size accounted for a significant amount of variation in the size of MCs.

Key words: body mass; dietary frugivory; digital dexterity; Meissner's corpuscles; primates.
\end{abstract}

\section{Introduction}

Meissner's corpuscles (MCs) are tactile mechanoreceptors found in the glabrous (i.e. hairless) skin of primates, including fingertips (Bolanowski \& Pawson, 2003; Hoffmann et al. 2004). They are localized in the dermal papillae (Fig. 1) and are characterized by sensitivity to light touch. MCs are rapidly adapting receptors that signal transient changes in pressure applied to the skin surface (Hoffmann et al. 2004; Organ et al. 2011; Zimmerman et al. 2014). As touch receptors, they provide high tactile acuity and are especially numerous in the fingertips (Johansson \& Vallbo, 1979; Caruso et al. 1994). Given their characteristics and distribution patterns, MCs have been likened to retinal photoreceptors in that they make up 'tactile fovea' of the fingers that

\section{Correspondence}

Andrey Verendeev, Department of Anthropology, The George Washington University, Washington, DC 20052, USA. T: 202.994.5923;

F: 202.994.6097; E: andrey.verendeev@gmail.com

Accepted for publication 13 April 2015

Article published online 5 June 2015 provides fine spatial resolution for cutaneous sensation (Hoffmann et al. 2004).

Functionally, MCs have been hypothesized to provide important sensory feedback for the effective control of grip (Purves et al. 2008). For example, Martin (1990) proposed that their association with papillary ridges enhances tactile perception and reduces slippage by allowing compensatory changes in the force of the grip (see also Preuss, 2009). This sensory control of grasping may have been especially important in the fine-branch niche of arboreal environments in which primates evolved. Others have suggested that MCs may aid in the assessment of fruit texture (Hoffmann et al. 2004). Overall, it has been suggested that MCs improve manipulative abilities in primates by providing important sensory feedback from the surface of the volar skin (Hoffmann et al. 2004).

Previous research has examined MCs in a number of primate species. For example, Winkelman (1963) published a comprehensive review describing MCs in apes, Old World monkeys, New World monkeys and strepsirrhine species. MCs were reported to be present in the glabrous skin of all primate species examined, but to be lacking in tree shrews, which are among the closest living relatives of primates. This 


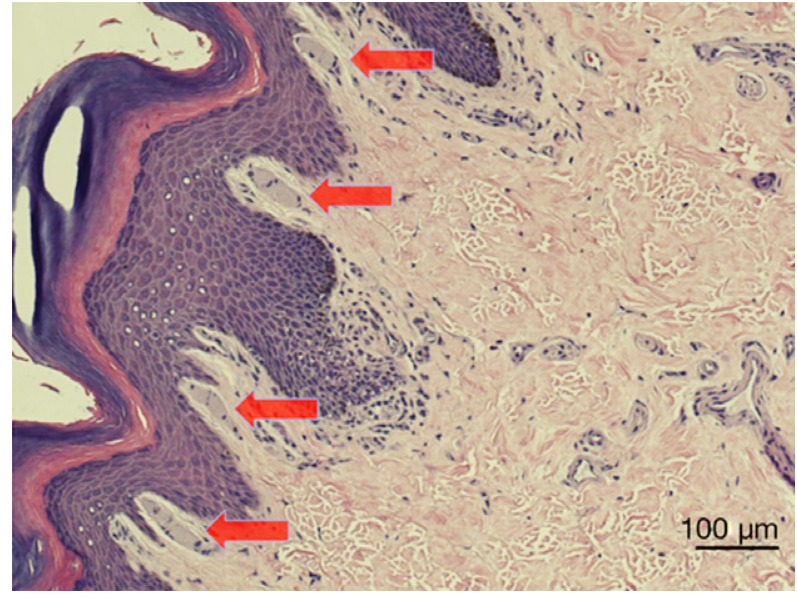

Fig. 1 MCs of a rhesus macaque (Macaca mulatta). MCs are indicated by red arrows.

led Martin (1990) to conclude that MCs likely evolved early in ancestral primates and were associated with adaptations to arboreal foraging. Despite their significance in primate evolution, it is notable that little comparative quantitative data exist on the distribution or size of MCs across primates.

Bolanowski \& Pawson (2003) examined the spatial organization and densities of MCs in rhesus monkey fingertips. The quantitative analysis was then extended to other monkey species (Güçlü et al. 2003) and apes (Hoffmann et al. 2004) for individual fingers and different hands. From these analyses it would appear that the average densities of MCs differ dramatically among primate species. For example, the density of MCs was reported to be 5.7 per $\mathrm{mm}^{2}$ for baboon (Güçlü et al. 2003) and 45.8 per $\mathrm{mm}^{2}$ for gibbon (Hylobates lar; Hoffmann et al. 2004). However, these studies differed considerably in their methodology, such as techniques of histological staining (e.g. cholinesterase vs. Masson's trichrome), sectioning procedures (e.g. transverse vs. longitudinal), and digits and/or number of sections per digit examined. Because these methodological differences may affect the estimates of density and size, a more systematic quantitative analysis of MCs in a range of primate species is warranted.

It has been previously argued that the evolution of prehension in primates involved independent control of digits (Bishop, 1964; Heffner \& Masterton, 1975; Napier, 1993), which may have evolved in the context of manipulation of small food items and other objects (Bishop, 1964; Jolly, 1970; Welles, 1976; Hoffmann et al. 2004). Primates differ in their manual ability, and several authors have attempted to rank primates in their manipulation skills according to a dexterity index developed by Heffner \& Masterton (1975) (see also Iwaniuk et al. 1999). Given the sensory characteristics of MCs, their role in the control of grip and the extent to which primates use their hands, it is possible that MCs may be associated with the evolution of manipulative abilities in primates, including the use of different grips in manipulation.
In the current study, we examined these relationships by analyzing MCs in six primate species, including humans (Fig. 2). We quantified the densities of MCs per $\mathrm{mm}^{2}$ and their size. Further, we examined whether MCs varied as a function of body mass, digital dexterity and dietary frugivory across the species in our sample. This allowed us to test the role of sensory feedback in the enhanced manual control of humans compared with other primates (Napier, 1961, 1993), as well as the fruit texture hypothesis of Hoffmann et al. (2004). Moreover, we examined how the density and the size of MCs were associated with the presence of the precision grip vs. power grip in the behavioral repertoire of the species. Specifically, given that the precision grip involves the use of the thumb in coordination with another digit (usually the index finger), we examined the distribution of MCs across fingers in relation to the ability to perform this grip type.

Another purpose of the present study was to examine how MCs may be related to handedness. Although hand preference is widespread and well documented in humans (Marchant \& Mcgrew, 1994; Raymond \& Pontier, 2004), it may not be restricted to our species (Mcgrew \& Marchant, 1997; Hopkins et al. 2007). For example, bias in hand use has been documented in chimpanzees (Hopkins et al. 2007) and other primate species (Fragaszy \& Mitchell, 1990; Westergaard \& Suomi, 1996; Spinozzi \& Truppa, 1999). Given that handedness involves one (i.e. dominant) hand performing the task with the other providing stabilizing support, we sought to examine whether this asymmetry in hand use (especially in humans) is reflected in the distribution patterns of MCs between the hands.

\section{Materials and methods}

\section{Subjects}

The non-human primate fingertip tissue was collected opportunistically at necropsy from specimens provided by Southwest National Primate Research Center (San Antonio, TX, USA), Yerkes National Primate Research Center (Atlanta, GA, USA) and the lowa Primate Learning Sanctuary (Des Moines, IA, USA). Fingertips from human cadavers were obtained from The George Washington University School of Medicine and Health Sciences. No animals were killed for the purpose of the present study. The specimens used are described in Table 1.

\section{Histological preparation}

Fingertips (i.e. skin sample from the digital pad) of the first (thumb), second (index) and fourth (ring) digits were collected from both hands of specimens, and stored in formalin solution. Fingertips were collected from these digits specifically to test the hypothesis of differential MC distribution in relationship to the use of the thumb and index finger in precision grip compared with other digits (i.e. ring finger). The fingertips were then embedded in paraffin, and the digital pad was dissected vertically into thin $(5 \mu \mathrm{m})$ trans- 

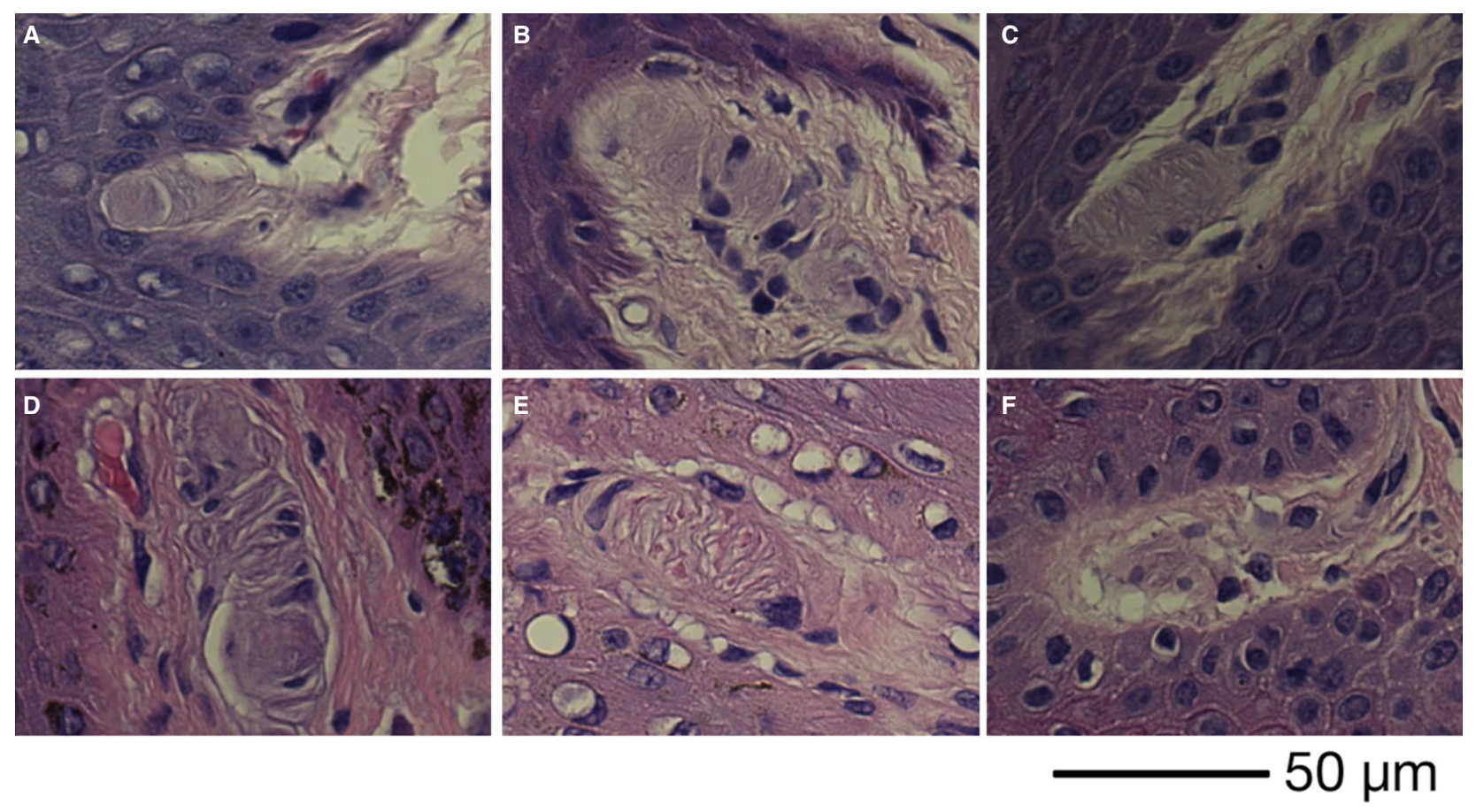

Fig. 2 MCs of Callithrix jacchus (A), Papio anubis (B), Macaca mulatta (C), Pan troglodytes (D), Pan paniscus (E) and Homo sapiens (F).

Table 1 Specimens used in the present study, including sex and age of each specimen when known.

\begin{tabular}{|c|c|c|c|c|c|c|c|}
\hline Species & Sex & Age & Not included & Species & Sex & Age & Not included \\
\hline \multirow{5}{*}{$\begin{array}{c}\text { Common marmoset } \\
\text { (Callithrix jacchus) }\end{array}$} & M & 2 & R1, R4 & \multirow{5}{*}{$\begin{array}{l}\text { Chimpanzee } \\
\text { (Pan troglodytes) }\end{array}$} & $\mathrm{F}$ & 24 & \\
\hline & M & 2 & R1 & & $\mathrm{F}$ & 57 & \\
\hline & M & 2 & & & $\mathrm{~F}$ & 50 & \\
\hline & M & 2 & L1, L2, L4 & & & & \\
\hline & M & 5 & L1 & & & & \\
\hline \multirow[t]{3}{*}{ Baboon (Papio anubis) } & $\mathrm{F}$ & 9 & & \multirow[t]{3}{*}{ Human (Homo sapiens) } & $\mathrm{F}$ & 78 & \\
\hline & M & 7 & & & $\mathrm{~F}$ & 89 & \\
\hline & & & & & M & 91 & \\
\hline \multirow{4}{*}{$\begin{array}{l}\text { Rhesus macaque } \\
\text { (Macaca mulatta) }\end{array}$} & $\mathrm{F}$ & 13 & L1 & & M & 85 & \multirow[t]{5}{*}{ L1, L2, L4 } \\
\hline & M & 20 & & & $\mathrm{~F}$ & $?$ & \\
\hline & $?$ & $?$ & R2 & & $\mathrm{F}$ & $?$ & \\
\hline & & & & & M & $?$ & \\
\hline Bonobo (Pan paniscus) & $\mathrm{F}$ & 44 & & & M & $?$ & \\
\hline
\end{tabular}

Not all fingertips were available for MCs quantification (due to poor quality tissue) and fingertips 'not' included in the analysis are listed in the 'Not Included' section of the table.

verse sections (3-4 per finger) in distal-proximal gradient and histologically stained using hematoxylin and eosin. The histological preparations were performed by Histo-Scientific Research Laboratories (HSRL, Jackson, VA, USA).

\section{Microscopy and data collection}

All tissue sections were coded prior to data collection to remove possible measurement bias. MCs were identified and quantified using a Zeiss Axioplan 2 photo-microscope equipped with a Ludl XY motorized stage (Ludl Electronic Products, Hawthorne, NY, USA), Heidenhain z-axis encoder, and an Optronics MicroFire color video camera (Optronics, Goleta, CA, USA) coupled to a Dell PC workstation running Stereolnvestigator software (MBF Bioscience, Williston, VT, USA). The length of the epidermis in the sample was measured along the surface of stratum granulosum using a $10 \times$ objective lens. MCs were quantified at a magnification of $20 \times$ along this length. The size of a corpuscle was measured as its cross-sectional diameter using Stereolnvestigator software (in $\mu \mathrm{m}$ ) by tracing a straight line across the approximate middle portion of each corpuscle and parallel to the surface of stratum granulosum. The average counts of MCs for each finger were determined by applying the Abercrombie correction formula (Abercrombie, 1946): $N=n *(T / T+H)$, where $n$ is the average number of MCs across all sections for the 
given fingertip, $T$ is the average section thickness, $H$ is the average size of MCs (averaged across all sections for the given fingertip) and $N$ is the corrected average number of MCs for each finger for the given length of epidermis. To estimate the average density of MCs (per $\mathrm{mm}^{2}$ ), we multiplied $N$ by $1 \mathrm{~mm}^{2}$ and divided it by the average area of epidermis measured for each fingertip calculated across all sections, the product being the average density of MCs per $\mathrm{mm}^{2}$ for each finger.

Several fingertips were excluded from the data collection due to poor quality of tissue (e.g. large portions of epidermis missing making it impossible to measure densities of MCs per $\mathrm{mm}^{2}$ of epidermal length). The excluded samples are detailed in Table 1; these were also excluded from the statistical analyses.

\section{Statistical analyses}

Because only one specimen of bonobo was available, MCs data for the bonobo and three chimpanzees were pooled and analyzed together as the genus Pan. One-way analyses of variance (ANOVA) were used to compare the density and size of MCs among the fingers and between the hands within each species. To compare MC densities and size among species, means were calculated across fingertips for each individual and these were used in cross-species comparisons using the non-parametric Kruskal-Wallis one-way ANOVA test. When appropriate, we used the Bonferroni correction for post hoc multiple comparisons. For comparisons between apes and monkeys, the data were pooled and analyzed together using an independent samples $t$-test. Ordinary least-squares regression analyses on species means were used to assess the relationship between MC densities or size and body mass, manual ability, and dietary frugivory. Digital dexterity scores found in Iwaniuk et al. (1999) were used as a proxy for manual ability. These scores were originally developed by Heffner \& Masterton (1975) on the basis of the anatomy of the hand described by Napier \& Napier (1967; see also Napier, 1961; e.g. opposable thumb vs. non-opposable thumb). Dietary frugivory was measured as the percentage of fruit in the diet including the feeding time, food intake or stomach content devoted to fruit, seeds or gums (Chivers, 1984). Previously published data on dietary frugivory were used (Clutton-Brock \& Harvey, 1977; Lindburg, 1977; Hubrecht, 1984, 1985). Humans were excluded from analyses of percentage of fruit consumption in the diet because meaningful data are not available. All significance levels were set at $\alpha=0.05$.

Data from closely related species do not satisfy the conditions of independence due to shared common ancestry, implicit in traditional parametric and non-parametric statistical tests (Iwaniuk et al. 1999; see also Felsenstein, 1985; Harvey \& Pagel, 1991). Therefore, we also performed phylogenetic generalized least-squares regressions (PGLS) using the caper package in R statistical software (Orme, 2013).

\section{Results}

Overall, we found no differences in $M C$ densities or size among the digits or between the hands for any species examined (Fig. 3). In other words, for any given species, MCs were similar in both their density and size across the digits and between the hands. In the ANOVA across species, omnibus Kruskall-Wallis one-way ANOVA showed significant species differences in both the density $\left[X^{2}(4)=13.717\right.$,
$P=0.008]$ and the size $\left[X^{2}(4)=18.620 ; P=0.001\right]$ of MCs. Bonferroni-adjusted post hoc pairwise comparisons revealed a trend for lower $\mathrm{MC}$ densities in genus Pan than in Homo sapiens $(P=0.099)$ and Macaca mulatta $(P=$ 0.068). In terms of MC size, Bonferroni-adjusted post hoc pairwise comparisons revealed significantly larger MCs in $H$. sapiens than in Callithrix jacchus $(P=0.001)$ and $M$. mulatta $(P=0.038)$; no other comparisons reached statistical significance (all $P>0.05$; Fig. 4).

To further explore phylogenetic variation, we compared MC densities and size between the apes (humans and chimpanzee species) and the monkeys (macaques, baboons and marmosets). Independent samples $t$-test revealed no differences in MC densities $\left(t_{20}=0.424 ; P=0.676\right)$ between these groups; however, apes had larger MCs than the monkeys $\left(t_{20}=8.650 ; P<0.001 ;\right.$ Fig. 5$)$.

It is possible that the observed species differences in the size of MCs reflect variation in body mass among the species in our sample. For example, the difference in body mass between chimpanzees and marmosets is over 100fold. To assess the effects of body mass on species differences in MCs, we examined the relationship between species mean body mass (data provided in Smith \& Jungers, 1997) and MC density or size using linear regression analyses. These analyses revealed a significant relationship between body mass and $\mathrm{MC}$ size $\left(R^{2}=0.917 ; F_{1,3}=33.054\right.$, $P=0.01)$, but not $\mathrm{MC}$ density $\left(R^{2}=0.020 ; \quad F_{1,3}=0.062\right.$, $P=0.819$; Fig. 6).

Next we examined MCs in relation to dexterity and dietary frugivory. Overall, regression analyses revealed no significant relationships between MC density or size and either dexterity or percentage of fruit consumption in $\operatorname{diet}$ (all $P>$ 0.05; see Fig. 7 for the summary of results).

We also performed analyses of the relationship between MCs data with body mass, dexterity score and percentage of fruit consumption in the diet using PGLS. Because there was no evidence for phylogenetic signal in our data set (all $\lambda=0$ ), the results of PGLS parallel those of ordinary leastsquares regression described above and are not reported here.

\section{Discussion}

Meissner's corpuscles are tactile mechanoreceptors found in the glabrous skin of primates. They are especially numerous in the fingertips (Johansson \& Vallbo, 1979; Caruso et al. 1994) and are characterized by sensitivity to movement across the skin (Zimmerman et al. 2014). Their association with papillary ridges has led to the hypothesis that MCs play a role in detection and reduction of slippage by allowing compensatory changes in the force of the grip (Martin, 1990; Preuss, 2009). This sensory control of grasping may have been especially important in the fine-branch niche of arboreal environments in which primates evolved. Additionally, MCs may aid in the evaluation of fruit texture, thus 

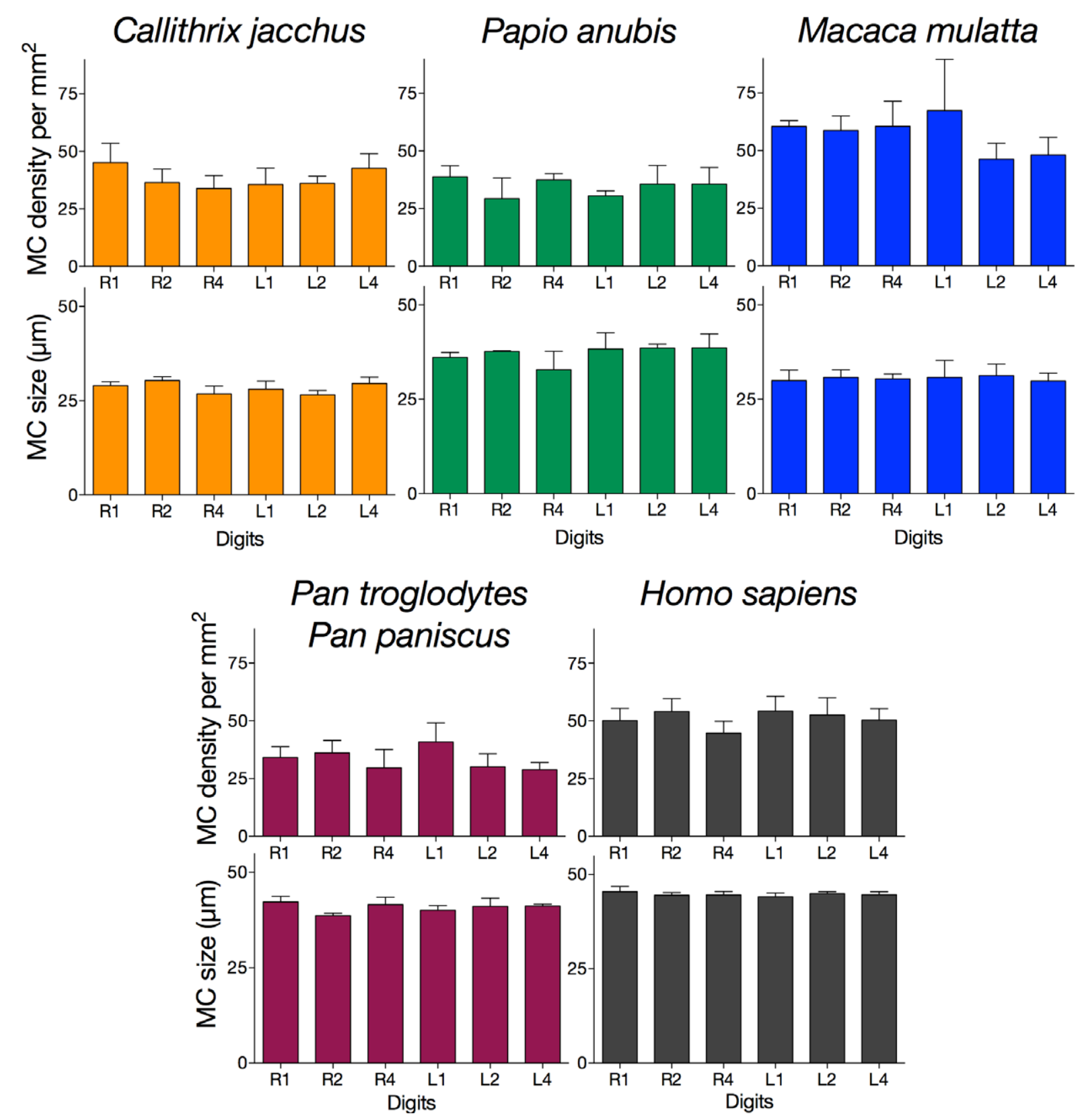

Fig. 3 Meissner's corpuscles (MCs) density per $\mathrm{mm}^{2}$ and MCs size across digits for each primate species. Error bars indicate SEM. One-way ANovA showed no differences in MCs density or size among the digits or between the hands for any species examined (all $P$ values non-significant). See text for more details.

allowing selection of ripe fruit (Hoffmann et al. 2004). Overall, it has been suggested that MCs improve manipulation abilities in primates by providing important sensory feedback from the surface of the hands and fingertips (Hoffmann et al. 2004).

The present study is a comparative quantitative analysis of MC density and size in different primate species. Previous quantitative analyses of MCs in primates used inconsistent histological staining techniques and counting methods, which sometimes resulted in data that are difficult to interpret. For example, Bolanowski \& Pawson (2003) measured the average density of MCs in rhesus monkey fingertips and found it to be about 45 per $\mathrm{mm}^{2}$. A later study by the same group, however, measured the average density of MCs for the same species to be 26.7 per $\mathrm{mm}^{2}$ (Güçlü et al. 2003). Because these differences can likely be explained on methodological grounds, in the present study we used a systematic approach to quantify MCs in several digits and both hands of specimens. Specifically, MC densities and size across digits and between hands were measured, and their relationship to precision grip, handedness, digital dexterity and dietary frugivory were assessed. 

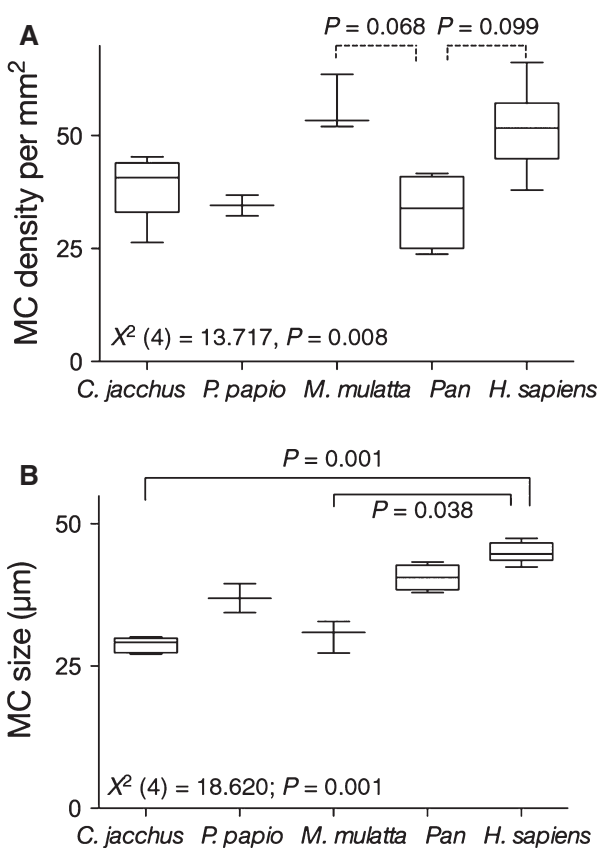

Fig. 4 Comparison of Meissner's corpuscles (MCs) density (A) and size (B) among the primate species. Box-plots indicate the 25 th percentile, the median and the 75th percentile; the whiskers indicate the range. Non-parametric Kruskall-Wallis one-way ANova showed significant species differences in both the density and the size of MCs. Bonferroniadjusted post hoc pairwise comparisons revealed significant differences between the species (solid line) or differences that approached statistical significance (dotted lines)

We found no differences in MC densities or size among digits or between hands for any species examined. This suggests no relationship between MCs and either the use of precision grip or handedness. First, among the species examined in our sample, all but the common marmoset (C. jacchus) are capable of employing precision grip in either the wild or captivity (Rose, 1977; Marzke \& Wullstein, 1996; Marzke, 1997; see also Napier, 1961); these species are also known for complex manipulation skills in relation to the use of feeding tools (Van Schaik et al. 1999; see also Torigoe, 1985). The precision grip usually involves the use of the thumb in association with another digit (usually the index finger; Napier, 1956; Landsmeer, 1962). No differences in MC distribution or size among digits were found, suggesting there is no relationship between variation in MCs across the digits and ability to perform the precision grip. It should be noted, however, that the precision grip is not a uniform behavior, and varies across species, maturity levels and object parameters (Marzke, 1997; Pouydebat et al. 2009). Precision grip is also largely determined by the anatomy of the hand. For example, chimpanzees and humans use different grips owing to different hand morphologies, reflecting differential proportion of thumb involvement relative to other digits. Nonetheless, assuming that the thumb is necessary for the 'true precision grip'
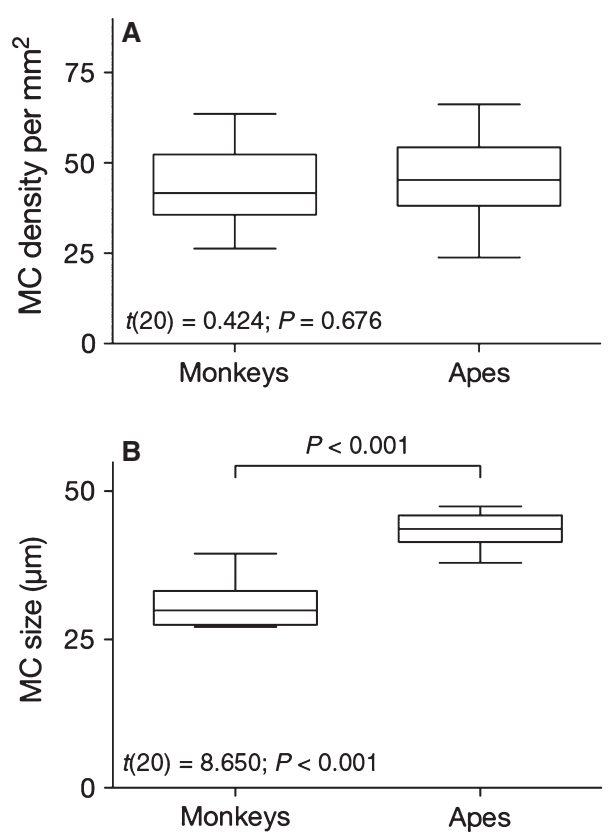

Fig. 5 Comparison of Meissner's corpuscles (MCs) density (A) and size (B) between apes (humans and two chimpanzee species) and monkeys (marmosets, baboons and rhesus). Box-plots indicate the 25th percentile, the median and the 75th percentile; the whiskers indicate the range. Independent samples $t$-test revealed a significant difference between these groups in MCs size but not density.

(Napier, 1961), and finding no differences in MC distribution between the thumb and other digits, the present data do not support a relationship between MCs and the use of the precision grip.

Second, the comparison of MCs between hands likewise revealed no differences between hands for any species examined, suggesting that there is no relationship between handedness and MC distribution. Although we do not know the handedness for our human specimens, assuming the well-recorded ratio of $9: 1$ right-handedness (Marchant \& Mcgrew, 1994; Raymond \& Pontier, 2004) in our sample, there is no evidence of a relationship between hand preference and MC distribution.

Comparison of MCs across all species revealed phylogenetic differences in MC density and size. Notably, humans had significantly larger MCs than marmosets and macaques. Overall, apes (humans and Pan species) had larger MCs than monkeys, although there was no difference in MC densities between the two groups. Given the extreme diversity in body mass among the species in the present study, we analyzed the relationship between body mass and MC size. Indeed, we found that body mass accounts for $92 \%$ of the variance of MC size in our sample. This increase in the size of MCs might be related to proportional increase in the size of the finger pads in larger animals.

It is interesting to note that macaque monkeys and humans had higher densities of MCs than other species. 

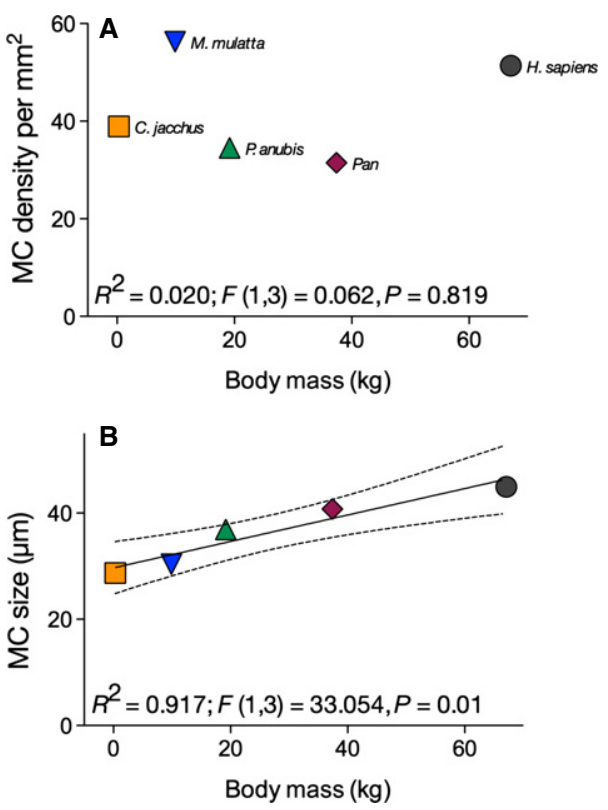

Fig. 6 Relationships between Meissner's corpuscles (MCs) density (A), size (B) and body mass $(\mathrm{kg})$. Body mass data are taken from Smith \& Jungers (1997). The regression line and the confidence intervals are derived from ordinary least-squares regression analysis. See text for more details.

Moreover, MC density was slightly higher (but not statistically significant) in rhesus macaques compared with humans. These results are consistent with previous reports showing higher densities per $\mathrm{mm}^{2}$ in rhesus macaques relative to humans (Bolanowski \& Pawson, 2003). It should also be noted, however, that MC density steadily declines with age (Cauna, 1956, 1965; Bolton et al. 1966; Iwasaki et al. 2003). All our human specimens were of advanced age; those of documented age were 78 years old or older. It is therefore possible that the density of MCs is higher in younger adults.

Regarding the lack of a relationship between MCs and either dexterity or dietary frugivory, we should point out a couple of important caveats. First, the dexterity score proposed by Heffner \& Masterton (1975) and later employed by Iwaniuk et al. (1999) was originally used for mammals at large. As a digital dexterity index, it was largely developed based on anatomical feature of thumb opposability (Napier \& Napier, 1967). As such, it may not accurately represent the more subtle variation in manipulative abilities across primates (for other criticisms of this dexterity index, see Heffner \& Masterton, 1975; Iwaniuk et al. 1999). Four out of six species in the present study received the score of 6 according to this dexterity index, with marmosets receiving 4 and humans a score of 7 . A different dexterity index based on behavioral observations developed specifically for primates is needed to more precisely characterize the diverse manipulative abilities of primate species (Torigoe, 1985).

Second, our species sample may have been too small to properly measure the relationship between MCs and dietary frugivory. With humans excluded from the analysis, our sample was effectively divided into two groups - Old World monkeys, and apes consuming $63-68 \%$ of fruit in their diet and marmosets whose diet mostly consists of fruit. With such a binary distribution, meaningful analyses are perhaps not possible. An additional limitation of the data on dietary frugivory used here is that a single measure may not adequately account for relevant population and intra-annual variability in fruit consumption in the wild. To address this issue, a larger interspecific sample with more diverse dietary habits would be needed.
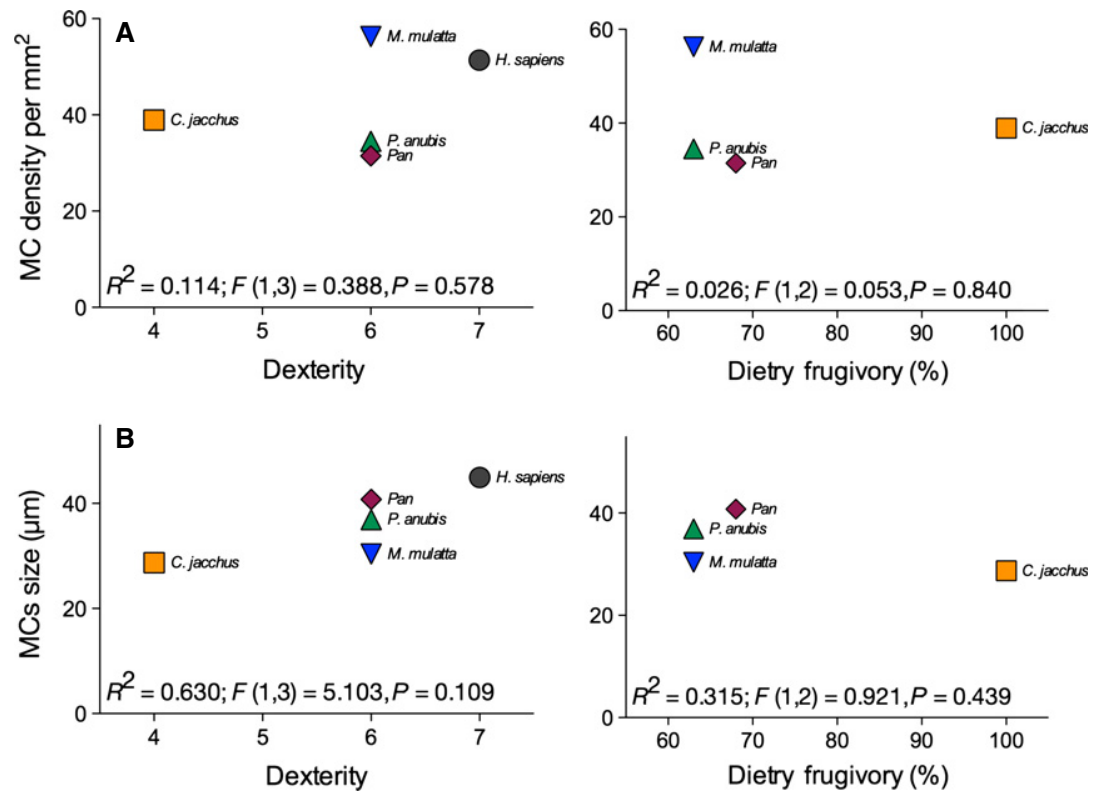

Fig. 7 Relationship between Meissner's corpuscles (MCs) density (A) and size (B), and either dexterity or dietary frugivory. The dexterity index was originally developed by Heffner \& Masterton (1975) on the basis of the anatomy of the hand described by Napier \& Napier (1967; see also Napier, 1961). The dietary frugivory was compiled from available data. See text for more details. 
The present study is the first to systematically examine MC densities and size in a number of primate species and across different digits and between hands of individuals. We also examined MC distribution in relation to a number of different behavioral characteristics. We show that $M C$ size, like other anatomical features, varies with body mass. Future studies should attempt to examine other tactile mechanoreceptors in relation to these variables.

\section{Acknowledgements}

The authors thank Dr Raymond Walsh for facilitating specimen collection from human cadavers. This work was supported by the National Institutes of Health (NS042867 and NS073134) and the James S. McDonnell Foundation (220020293).

\section{References}

Abercrombie M (1946) Estimation of nuclear population from microtome sections. Anat Rec 94, 239-247.

Bishop A (1964) Use of the hand in lower primates. In: Evolutionary and Genetic Biology of Primates, Vol. 2. (ed. BuettnerJanusch J), pp. 133-225. New York: Academic Press.

Bolanowski SJ, Pawson L (2003) Organization of Meissner corpuscles in the glabrous skin of monkey and cat. Somatosens Mot Res 20, 223-231.

Bolton CF, Winkelmann RK, Dyck PJ (1966) A quantitative study of Meissner's corpuscles in man. Neurology 16, 1-9.

Caruso G, Nolano M, Lullo F, et al. (1994) Median nerve sensory responses evoked by tactile stimulation of the finger proximal and distal phalanx in normal subjects. Muscle Nerve 17, 269275.

Cauna N (1956) Nerve supply and nerve endings in Meissner corpuscles. Am J Anat 99, 315.

Cauna N (1965) The effects of aging on the receptor organs of the human dermis. In: Advances in Biology of Skin, Vol. VI: Aging. (ed. Montagna W), pp. 63-96. New York: Pergamon.

Chivers DJ (1984) A summary of feeding and ranging in Gibbons. In: The Lesser Apes. (eds Preuschoft $\mathrm{H}$, Chivers DJ, Brockelman WY, Creel N), pp. 267-281. Edinburgh: Edinburgh University Press.

Clutton-Brock TH, Harvey PH (1977) Species differences in feeding and ranging behaviour in primates. In: Primate Ecology. (ed. Clutton-Brock TH), pp. 557-584. London: Academic Press.

Felsenstein J (1985) Phylogenies and comparative method. Am Nat 125, 1-15.

Fragaszy DM, Mitchell SR (1990) Hand preference and performance on unimanual and bimanual tasks in capuchin monkeys (Cebus apella). J Comp Psychol 104, 272-282.

Güçlü B, Bolanowski SJ, Pawson L (2003) End-to-end linkage (EEL) clustering algorithm: a study on the distribution of Meissner corpuscles in the skin. J Comput Neurosci 15, 19-28.

Harvey PH, Pagel MD (1991) The Comparative Method in Evolutionary Biology. Oxford: Oxford University Press.

Heffner R, Masterton B (1975) Variation in form of the pyramidal tract and its relationship to digital dexterity. Brain Behav Evol 12, 161-200.

Hoffmann JN, Montag AG, Dominy NJ (2004) Meissner corpuscles and somatosensory acuity: the prehensile appendages of primates and elephants. Anat Rec 281, 1138-1147.
Hopkins WD, Russell JL, Lambeth S, et al. (2007) Handedness and neuroanatomical asymmetries in captive chimpanzees: a summary of 15 years of research. In: Evolution of Hemispheric Specialization in Primates. (ed. Hopkins WD), pp. 146-181. Oxford: Elsevier.

Hubrecht RC (1984) Field observations on group size and composition of the common marmoset (Callithrix jacchus jacchus) at Tacapura, Brazil. Primates 25, 13-21.

Hubrecht RC (1985) Home range size and use and territorial behaviour in the common marmoset (Callithrix jacchus jacchus) at the Tacapura field station, Recife, Brazil. Int J Primatol 6, 533-550.

Iwaniuk AN, Pellis SM, Whishaw IQ (1999) Is digital dexterity really related to corticospinal projections? A re-analysis of the Heffner and Masterton data set using modern comparative statistics. Behav Brain Res 101, 173-187.

Iwasaki T, Goto N, Goto J, et al. (2003) The aging of human Meissner's corpuscles as evidenced by parallel sectioning. Okajimas Folia Anat. Jpn 79, 185-190.

Johansson RS, Vallbo AB (1979) Tactile sensibility in the human hand: relative and absolute densities of four types of mechanoreceptive units in glabrous skin. J Physiol 286, 283-300.

Jolly CJ (1970) The seed-eaters: a new model of hominid differentiation based on a baboon analogy. Man 5, 5-26.

Landsmeer J (1962) Power grip and precision handling. Ann Rheum Dis 21, 164-170.

Lindburg DG (1977) Feeding behavior and diet of rhesus monkeys (Macaca mulatta) in a Siwalik Forest in North India. In: Primate Ecology: Studies of Feeding and Ranging Behaviour in Lemurs, Monkeys and Apes. (ed. Clutton-Brock TH), pp. 223249. London: Academic Press.

Marchant LF, Mcgrew WC (1994) Human handedness: an ethological perspective. Hum Evol 13, 221-228.

Martin R (1990) Primate Origins and Evolution: a Phylogenetic Reconstruction. Princeton, NJ: Princeton University Press.

Marzke MW (1997) Precision grips, hand morphology, and tools. Am J Phys Anthropol 102, 91-110.

Marzke MW, Wullstein KL (1996) Chimpanzee and human grips: a new classification with a focus on evolutionary morphology. Int J Primatol 17, 117-139.

Mcgrew WC, Marchant LF (1997) On the other hand: current issues in and metaanalysis of the behavioral laterality of hand function in non-human primates. Yearb Phys Anthropol 40, 201-232.

Napier JR (1956) The prehensile movements of the human hand. $J$ Bone Joint Surg 38B, 902-913.

Napier JR (1961) Prehensility and opposability in the hands of primates. Symp Zool Soc Lond 5, 115-132.

Napier JR (1993) Hands. Princeton, NJ: Princeton University Press.

Napier JR, Napier PH (1967) A Handbook of Living Primates. New York: Academic Press.

Organ JM, Muchlinski MN, Deane AS (2011) Mechanoreceptivity of prehensile tail skin varies between ateline and cebine primates. Anat Rec 294, 2064-2072.

Orme D (2013) The caper package: comparative analysis of phylogenetics and evolution in R. $R$ package version $0.5,2$.

Pouydebat E, Gorce P, Coppens Y, et al. (2009) Biomechanical study of grasping according to the volume of the object: human versus non-human primates. J Biomech 42, 266-272.

Preuss TM (2009) Primate brain evolution. In: Evolutionary Neuroscience. (ed. Kaas JH), pp. 793-825. Oxford: Academic Press. 
80 Meissner's corpuscles in primate fingertips, A. Verendeev et al.

Purves D, Augustine GJ, Fitzpatrick D, et al. (2008) Neuroscience, 4th edn. Sunderland, MA: Sinauer.

Raymond M, Pontier D (2004) Is there geographical variation in human handedness? Laterality 9, 35-51.

Rose MD (1977) Positional behaviour of olive baboons (Papio anubis) and its relationship to maintenance and social activities. Primates 18, 59-116.

Smith RJ, Jungers WL (1997) Body mass in comparative primatology. J Hum Evol 32, 523-559.

Spinozzi G, Truppa V (1999) Hand preferences in different tasks by tufted capuchins (Cebus apella). Int J Primatol 20, 827-849.

Torigoe T (1985) Comparison of object manipulation among 74 species of non-human primates. Primates 26, 182-194.
Van Schaik CP, Deaner RO, Merrill MY (1999) The conditions for tool use in primates: implications for the evolution of material culture. J Hum Evol 36, 719-741.

Welles JF (1976) A comparative study of manual prehension in anthropoids. Saugetierlaundliche Mitteilungen 24, 26-38.

Westergaard GC, Suomi SJ (1996) Hand preference for a bimanual task in tufted capuchins (Cebus apella) and rhesus macaques (Macaca mulatta). J Comp Psychol 110, 406-411.

Winkelman RK (1963) Nerve endings in the skin of primates. In: Evolutionary and Genetic Biology of Primates. (ed. BuettnerJanusch J), pp. 229-259. New York: Academic Press.

Zimmerman A, Bai L, Ginty DD (2014) The gentle touch receptors of mammalian skin. Science 346, 950-954. 